\title{
The Application and Improvement of S-P Chart Method in Teaching Evaluation
}

\author{
Hongwei Ding* \\ School of Information Science and Engineering \\ Yunnan University \\ Kunming, China \\ Bo Li \\ School of Information Science and Engineering \\ Yunnan University \\ Kunming, China
}

\author{
Zongshan Wang \\ School of Information Science and Engineering \\ Yunnan University \\ Kunming, China \\ Liyong Bao \\ School of Information Science and Engineering \\ Yunnan University \\ Kunming, China
}

\author{
Jundong Yang \\ Information Science and Engineering \\ Yunnan University \\ Kunming, China
}

\begin{abstract}
In order to meet the higher requirements of examination evaluation put forward by the educational reform in the new period, it is particularly important to introduce scientific and systematic evaluation methods into examination evaluation. Based on formative evaluation and the combination of qualitative and quantitative research, S-P chart evaluation method provides a fair, objective and comprehensive evaluation of test scores. It is favored by many educators and researchers for its graphical data expression and strong data processing ability, and has been widely used in various fields and various types of test evaluation. However, there are still some unsatisfactory aspects when people understand and use the $\mathbf{S}-\mathbf{P}$ chart, such as the operation is more complicated, the evaluation parameters are not accurate and so on. This paper first introduces the main functions, advantages and disadvantages, applications and research status of S-P chart at home and abroad, then systematically combs several improvement methods of S-P chart, and finally looks forward to the future development direction of S-P chart.
\end{abstract}

Keywords -S-P chart; evaluation method; improved; method

\section{INTRODUCTION}

With the full implementation of the new round of curriculum reform and the development of teaching quality evaluation, examination quality analysis has been paid more and more attention by front-line teachers, teaching administrators and educational researchers. As an educational evaluation method, examination has the functions of testing students' academic level and teachers' teaching effect, feedback teaching information, identifying talents and so on.

This work was supported in part by the National Science Foundation of China named Research on Theory and Control Protocol of Convergence Multiple Access Communication Network (61461053), and in part by the National Science Foundation of China named Research on Analysis and Improvement of Polling Control System in Wireless Network (61461054), and in part by the National Science Foundation of China named Research on Theoretical Evolution of Polling System and Application Control Protocol (61072079)
The traditional examination evaluation is often just a simple statistics of the examination results, which is used to measure the students' ability. This method of analysis of the examination results puts too much emphasis on objectification, standardization and quantification. Thus, it ignores those important qualities and behaviors that are difficult to quantify, and ignores personality development and multiple standards. The original rich personality psychological development and behavior performance are simplified into abstract score representation and quantitative calculation. Therefore, the new round of curriculum reform proposes to increase the analysis of students" "quality" on the basis of the traditional quantitative evaluation, so as to change the examination evaluation from the symbolic score mechanism to the substantive content mechanism.

\section{S-P CHART AND ITS MAIN FUNCTIONS}

\section{A. Brief Introduction of S-P Chart}

In order to diagnose the students' learning situation and decide the individual tutoring content, a Japanese scholar put forward a numerical quantitative test graphic analysis method, which is called Student-Problem Chart. It is a cognitive diagnostic average technique developed by combining cognitive theory and test theory to graphically describe students' responses to test questions. Using S-P chart as the tool of measurement and evaluation, an active teaching management system is established, the results of teaching tests are analyzed and diagnosed with both quality and quantity, and the reference basis for evaluating students and improving teaching quality is put forward. Compared with the traditional evaluation methods of calculating total score, ranking and judging passing rate, it provides more feedback information, 
which means that the test can really play the function of evaluation.

\section{B. Main Functions of S-P Chart}

At present, the vast majority of the evaluation of examinations in primary and secondary schools in China only judge the position of students in the class by ranking scores and comparing students' scores, and analyze their learning level and learning status. The resulting conclusions are often too simple and one-sided, and it is not easy to accurately reflect the students' cognitive level and stability as well as the overall tendency of the class. This is very disadvantageous to the objective evaluation of students, the implementation of teaching and remedial work. S-P chart is different from the traditional evaluation method, it not only improves the quantitative standard of evaluation, but also can qualitatively evaluate the results. It is a combination of quantity and form evaluation method. According to the demonstration of its inventors, it is pointed out that the function of S-P chart in teaching evaluation mainly has the following five items:

1. The processing and analysis of the S-P chart is simple and can be done by any teacher.

2. The interpretation of the S-P chart is clear at a glance, not only to grasp the situation of the whole class, but also to interpret individual students and individual examination questions.

3. In terms of learning and diagnosis, the function of S-P chart can be used for both "quantitative analysis" and "qualitative analysis". Its function is practical and extensive.

4. The results of S-P chart analysis can provide improved data and clues for teaching, tutoring and asking questions.

5. The S-P chart analysis can be used not only in the analysis of test results and test questions, but also in the analysis of pre-test and post-test.

\section{ThE AdVANTAGES AND DiSADVANTAGES OF S-P CHART}

\section{A. The Advantages of S-P Chart}

- The S-P chart evaluation method uses a certain standard to change the students' examination scores into " 0,1 ", which makes the original complicated data simple and easy to deal with.

- The graphical analysis method given by the S-P chart evaluation method makes the law of the original data intuitive and avoids the defect of too complicated calculation in the past.

- The analysis theory of the S-P chart evaluation method is higher than the traditional examination analysis theory, paying attention to the combination of quantitative and qualitative analysis, the formation of the evaluation process and the development of the evaluation conclusion.

- The evaluation method of S-P chart includes the indexes commonly used in the statistics of test scores, such as the highest score, the lowest score, the passing rate, the average score, the standard deviation, the frequency distribution of scores, etc., as well as its own unique qualities, such as S-P curve, difference, stability coefficient, suitability coefficient, warning parameters and so on.

- The statistics and processing of data by S-P chart evaluation method can basically be completed by Excel, which is easy to popularize and use.

- The S-P chart evaluation method is not only designed for large-scale examinations, it is more suitable for all kinds of tests and examinations in the teaching stage, and the guiding function of test evaluation is applied to every link of curriculum implementation.

\section{B. The Disadvantages of S-P Chart}

While the S-P chart evaluation method has many advantages, it also has many shortcomings. The S-P chart evaluation method classifies students' learning types roughly, but divides students' learning types into six types, and cannot point out in detail the shortcomings in students' learning, that is, when students can't point out whether they really master a certain concept or some learning materials, and carry out formative evaluation of classes with a small number of students It is more appropriate to use the S-P chart. In view of the shortcomings of the S-P chart evaluation method, many improved methods came into being.

\section{RELATED LITERATURE REVIEW}

In reference [1], aiming at the problem that S-P chart evaluation method is only suitable for classes with a small number of students, this paper discusses how to make use of the advantages of modern cognitive diagnostic measurement theory to combine modern cognitive diagnostic measurement theory and technology with S-P chart method to provide more accurate and effective feedback information, and strive to use this method in situations with a large number of people. The basic idea of improving the S-P chart method is to absorb the relevant knowledge of the modern cognitive diagnosis theory on the basis of the classical measurement theory without the help of the complex psychometric model, so as to achieve the purpose that the later classification of the S-P chart table method is too rough.

Combined with the relevant knowledge of cognitive diagnosis theory, the basic steps for the improvement of S-P chart method are as follows:

1. Determine the cognitive attributes of the content to be tested and the hierarchical relationship between the attributes.

2. According to step (1), the reachable matrix $R$ and the subject $\mathrm{Q}$ matrix are given, and the test blueprint is designed.

3. The students' total test scores and observation response patterns (ORP) were analyzed by S-P chart method.

4. The ORP, of any subject $i$ is recorded as $\operatorname{ORP}(i)$, According to the standard error of the test, the confidence interval of $\left[a_{i}, b_{i}\right]$ the total score of $\operatorname{ORP}(i)$ is calculated. 
5. Look for all the IPR whose total score falls in the interval, $\left[a_{i}, b_{i}\right]$ and the set of these IRP is marked as $\operatorname{IRPS}_{i}$.

6. The Mahalanobis distance method is used to find an IRP, from IRPS $i$ to minimize the distance between it and ORP $(i)$. The Mahalanobis distance between ORP and IRP is determined by the inverse of the variance matrix of the observed score of the corresponding item.

7. According to the ideal response model obtained in step (6), the student's knowledge state (that is, attribute mastery mode) can be known.

8. Repeat steps (4) through (7) until all the subjects' KS are obtained

9. The synthesis of S-P chart analysis and KS derived from step (3) to step (8) is to improve the results of S-P chart analysis.

However, when using this method, the following issues are worth paying attention to:

1. The importance of determining attributes and attribute hierarchical relationships.

2. The influence of diagnostic classification methods on diagnostic classification.

\section{Improve the limitation of S-P chart method.}

In view of the above problems, on the basis of summing up the practical experience, literature [2] has made the following two improvements on the use of S-P chart analysis: one is to cooperate with qualitative evaluation, so that the scope of application of S-P chart is wider and more in line with the evaluation law; the other is to computerize the operation of S-P chart, so that the complex analysis can be automated.

In the first improvement, the paper not only fully observed the data distribution in the S-P chart, but also combined with the students' own analysis, but also took into account the qualitative judgment of teachers. The application of S-P chart analysis not only grasps a certain relationship of things themselves, but also the strict stipulation of mathematics is also helpful for us to obtain an accurate description of something, and the principles and rules of mathematics can also promote our further thinking, so that we can gain more profound understanding and new discoveries by virtue of mathematical reasoning. At the same time, the comprehensive use of students' self-evaluation and teachers' qualitative evaluation can get rid of the limitation of quantitative relationship, so as to grasp the "truth" more clearly.

The second improvement of S-P chart analysis in this paper is to realize the computerization of the analysis process. This improvement is actually a technical optimization of S-P chart analysis itself. This paper proposes that we can use VBA (Visual Basic for Application), the design language of macro processing in Excel, to compile the S-P chart analysis program based on Excel, so as to realize the automation of the analysis process. In concrete use, as long as the answers of the students and the standard answers of the test questions are entered into the Excel table, and then the macro program compiled by VBA is executed, the analysis results of the S-P chart automatically generated by the program can be obtained. In addition to easily obtaining statistical indicators such as "difference coefficient" and "attention coefficient", the analysis results of each student and each question can also be automatically placed in the student category diagnosis table. Practice has proved that this is completely feasible and very convenient and practical.

The research of data processing and analysis on computer has existed for a long time, but the software that can really be used for test score analysis is not very popular, and a considerable part of the reason is that teachers do not attach importance to the analysis of test results. In addition, some practical situations in life also lead to the lack of application in $\mathrm{S}-\mathrm{P}$ chart. This makes it necessary to design a S-P chart software.

The purpose and significance of designing S-P chart software are as follows:

\section{The significance of examination score Analysis}

2. It is of great practical significance to evaluate the test papers.

Based on the above analysis, reference [3] puts forward a software design process of S-P chart, which is designed and implemented by Excel based on the scientific theory and method of educational measurement and evaluation.

The idea of software design has the following three aspects:

1. Try to be more concise and convenient.

2. It is widely and universally used.

3. The tools used.

During the design period, the implementation process of the software and the problems encountered are as follows:

1. Realize the sorting problem of S-P chart from top to bottom and from left to right.

2. The problem of automatic drawing of S-line and P-line.

The research on the application of S-P chart evaluation method is to make students" grades " 0 or 1 " according to a certain standard, which is very effective for analyzing objective problems, but it reduces some information about students' scores. In order to better solve the above problems, literature [4] proposes to introduce the theory of fuzzy mathematics into S-P chart to form the theory of fuzzy S-P chart method, which can better evaluate the degree of students' correct answer in scoring questions. The improved S-P chart evaluation system makes use of the macro command function of Excel, and absorbs the idea of fuzzy S-P chart according to the analysis method and steps of the traditional S-P chart, and designs three functional modules: test paper information collection, S-P chart automatic processing and data analysis.

This paper uses a specific example to further illustrate the improved S-P chart method. Through the examples, it can be concluded that the S-P chart analysis and evaluation system developed based on Excel VBA has a wider analysis scope, more practical, and simple operation. But at the same time, it has some limitations. For example, it can only use S-P chart to analyze according to a topic and a unit, so it is not suitable for 
the analysis of large sample data. It can deal with and analyze objective questions, multiple choice questions and score questions. However, this promotion cannot be applied to multiple choice questions and so on. In view of the limitations of the small sample analysis of the S-P chart, teachers can adopt a one-to-one corresponding way, that is, a S-P chart corresponds to a project evaluation of a class of students, so that it is both organized and clear at a glance. The limitation of multiple choice questions in the analysis of S-P chart mainly lies in the diversity of students' scores of multiple choice questions and their responses to the choice of questions. If teachers do not need to understand the distribution of students' responses to question options, they can deal with them as scoring questions. teaching evaluation is a systematic and complicated project. At present, the classification standards of students' learning types and problem types given by the S-P chart evaluation system are based on previous studies, and the further refinement of the classification criteria needs to be further studied.

\section{THE APPLICATION OF S-P CHART}

In recent years, with the new round of curriculum reform, it has a far-reaching impact on the improvement of education and teaching effect. The application of S-P chart is becoming more and more popular, and the application scenarios are also increasing. Reference [5] describes the application of S-P chart analysis in online learning. As the name implies, online learning, that is, learning on the Internet, using standard Internet technology as a medium to transmit and interact with learners and learning resources, learning and can provide a lot of coordination and interaction. The evaluation method of S-P chart is applied to online learning in order to understand the students' use of online learning and the appropriateness of using the test questions bank drawn up by students themselves. Classroom learning with online learning is adopted as the way of course learning, and each student draws up the test questions in the final stage of the course to explore whether the students' learning attitude, adaptation and this teaching strategy are appropriate.

T-P chart is generally used for teaching evaluation. Literature [6] introduces the use of S-P chart in educational information processing, in order to further improve the evaluation method of information-based examination. This paper uses the S-P chart to analyze the results of English teaching evaluation in order to understand the learning behavior of individual students, the heterogeneity of individual test questions, the learning effectiveness of the whole class and the quality of the overall test. Using the S-P chart as the analysis tool of learning evaluation, this paper makes a qualitative and quantitative analysis and diagnosis of the results of teaching evaluation, and puts forward some reference information for improving English teaching and test quality. For the traditional scoring, ranking test evaluation, to provide more feedback information, so that the test can really play the role of evaluation, to achieve effective teaching.
In recent years, with the promotion and application of multimedia computer technology, multimedia teaching has been generally welcomed by the majority of teachers and students, and more and more subject teachers have joined the ranks of making multimedia courseware. What is the actual effect of multimedia teaching and how to evaluate it? This has become the focus of attention. For this reason, reference [7] compares the commonly used multimedia production software, and then compiles the S-P chart analysis software program with ASP language to evaluate the teaching effect.

\section{CONCLUSION}

As mentioned above, the evaluation of S-P chart is very promising. However, this development also requires the joint efforts of everyone to enrich. On the premise of computer automatic processing, the S-P chart can be used as the basis of test paper and examination evaluation. On the one hand, the original data table of S-P chart is the most complete for the collection of examination data, including the score of each small question, so it is convenient to expand its application on it; on the other hand, S-P chart also has its unique processing method, which can make a simple and effective evaluation of the whole test, which is difficult to do by other evaluation theories. Therefore, we can make efforts from the following aspects.

- Enrich the evaluation theory.

- Enrich the content of evaluation.

- Evaluate the universality of application.

- The meta-evaluation theory needs to be further developed.

- $\quad$ Perfect the evaluation procedure.

\section{REFERENCES}

[1] Z. Xu, S. Ding, Q. Yang, "The Improved for S-P Chart Method and Application," Journal of Jiangxi Normal University (Natural Science Edition), vol. 35, no. 5, pp. 543-547, 2011. (In Chinese).

[2] J. Wang, "Application and Improvement of S-P Chart Analysis" Educational Measurement and Evaluation, vol. 11, pp. 6-11, 2009. (In Chinese).

[3] Z. Hong "Software Design of S-P Chart," Science and Technology Information, vol. 25, 2008. (In Chinese).

[4] Y. Ou, "Improvement and Application of Excel VBA-based S-P Char Evaluation System-Taking a Physics Test as an Example," Physics Bulletin, vol. 12, pp. 114-118, 2013. (In Chinese).

[5] Y. Huang, S. Weng, Z. Shi, "S-P Table in E-Learning," Journal of Shenzhen Polytechnic, vol. 8, no. 06, 2009. (In Chinese).

[6] Q. Gan, "Application of S-P Chart in Teaching Evaluation," Software Guide, vol. 11, no. 09, pp. 76-78, 2012. (In Chinese)

[7] Y. Luo, "The application of S-P Chart analysis in multimedia teaching evaluation," Management Observer, vol. 18, pp. 169-170, 2009. (In Chinese). 